\title{
SOME GENERAL THEOREMS ON THE COHOMOLOGY OF CLASSIFYING SPACES OF COMPACT LIE GROUPS
}

\author{
BY \\ MARK FESHBACH ${ }^{1}$
}

\begin{abstract}
This paper is divided into two parts. The first part proves a number of general theorems on the cohomology of the classifying spaces of compact Lie groups. These theorems are proved by transfer methods, relying heavily on the double coset theorem [ $\left.F_{1}\right]$. Several of these results are well known while others are quite new. For the most part the proofs of the theorems are independent of each other and are quite short. Nevertheless they are true in great generality. Several are proven for arbitrary compact Lie groups and arbitrary cohomology theories. Perhaps the most interesting of the new results relates the cohomology of the classifying space of an arbitrary compact Lie group with that of the normalizer of a maximal torus.

The second part of the paper generalizes many theorems to certain equivariant cohomology theories. Some of these theorems appear in $\left[\mathbf{F}_{\mathbf{2}}\right]$.
\end{abstract}

I. Let $h$ be any general cohomology theory in the sense of [Dy]. As is well known, a map $\rho: X \rightarrow Y$ induces a homomorphism $\rho^{*}: h(Y) \rightarrow h(X)$. In certain situations a transfer homomorphism $t^{*}: h(X) \rightarrow h(Y)$ exists also [BG], $\left[\mathbf{D}_{0}\right]$. In this paper $G$ is an arbitrary compact Lie group. $H$ and $K$ are closed subgroups of $G$. $T$ is a maximal torus of $G$ with normalizer $N$. We shall refer of ten to [ $\left.\mathbf{F}_{0}\right]$ for notation. If $\rho(H, G): B H \rightarrow B G$ is the natural projection of classifying spaces, a transfer homomorphism exists, $T(H, G): h(B H) \rightarrow h(B G)$ [F, II.5]. (Technically $T(H, G)$ is only defined for finite-dimensional approximations of $\rho(H, G)$ (Definition I.6) but this restriction should be overcome in the near future. Until this restriction is removed all the theorems quoted in this paper will technically only be proven for the finite-dimensional approximations of $\left.\rho(H, G){ }^{2}\right) h_{S}$ is stable cohomotopy. This theory, represented by the sphere spectrum, acts on all others via the smash product.

We use two properties of the transfer to prove various theorems relating $h(B G)$ and $h(B H)$ for arbitrary cohomology theories.

The following is the most famous property of the transfer. We state it in a slightly different form than usual to facilitate its applications.

ProperTy I.1. Let $B$ be a paracompact and locally compact connected space. Let $\pi$ : $Y \rightarrow B$ be a locally trivial fibration with fiber $F$ a compact manifold. Then a transfer exists $t^{*}: h(Y) \rightarrow h(B)$ and $t^{*} \circ \pi^{*}$ is multiplication by the universal fixed point index

Received by the editors July 12, 1978 and, in revised form, March 6, 1980.

1980 Mathematics Subject Classification. Primary 55R40; Secondary 55N20, 57S15.

${ }^{1}$ Research was partially supported by a grant from NSF.

${ }^{2}$ Peter May has informed me that Gaunce Lewis has accomplished this. I also understand that $\mathbf{M}$. Prieto's thesis extends the definition of the transfer correctly. 
$\chi(F)+u \in h_{s}^{0}(B)$. Furthermore $u \in \tilde{h}_{s}^{0}(B)$. If $h=H^{*}$ is singular cohomology $t^{*} \circ \pi^{*}$ is multiplication by $\chi(F)$. (Although not stated in this form Property I.1 follows from the following papers, $[\mathbf{B M}],\left[\mathbf{D}_{0}\right],\left[\mathbf{D}_{2}\right]$. It is true in even more generality than stated.)

This property is quite powerful by itself. For example it is an easy exercise to prove from it that $H^{*}(B G, Q) \simeq Q$ for $G$ finite. (Let $H$ equal the trivial subgroup of $G$ and consider the fibration $\rho(H, G)$.)

The next property is much more complicated. However it simplifies in many special circumstances. Let $K|G| H$ be the double coset space obtained as the orbit space of the left action of $K$ on $G / H$. This space breaks up into a finite disjoint union of orbit-type manifold components $\{M\}$. Let $g \in G$ be a representative of $M$. Let $\chi^{\#}(M)=\chi(\bar{M})-\chi(\bar{M}-M)$ be the internal Euler characteristic of $M$ (e.g., $\chi^{\#}$ (point) $=1, \chi^{\#}$ (interior of a line segment) $=-1$ ). Let $H^{8}=g \mathrm{Hg}^{-1}$ and let $C g: h(B H) \rightarrow h\left(B H^{g}\right)$ be the usual conjugation isomorphism. This is induced by $\tilde{C} g: B H^{g} \rightarrow B H\left[F_{0}\right.$, II.3]. (See also Definition II.1 of this paper when $X$ equals a point.) We have the following.

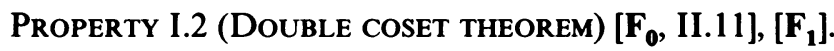

$$
\rho^{*}(K, G) \circ T(H, G)=\sum \chi^{\#}(M) T\left(H^{g} \cap K, K\right) \circ \rho^{*}\left(H^{g} \cap K, H^{g}\right) \circ C g
$$

where the sum is over the orbit-type manifold components $\{M\}$ of the double coset space $K|G| H$.

This theorem holds for arbitrary cohomology theories where $G$ is a compact Lie group and $H$ and $K$ are arbitrary closed subgroups of $G$. The formula is quite complicated. However, as we shall see, it simplifies nicely in a number of circumstances.

We use the following several times.

Corollary I.3 [F, VI.3]. Let $G$ be a compact Lie group and $H$ any closed subgroup of $G$. Let $K$ be a torus in $G$. Then

$$
\rho^{*}(K, G) \circ T(H, G)=\sum \chi^{\#}(M) \rho^{*}\left(K, H^{g}\right) \circ C g
$$

where the sum is over the manifold components $\{M\}$ of the fixed point orbits of $K$ in $K|G| H$ where $g \in G$ is a representative of $M$.

If $T=H=K$ is a maximal torus of $G$, then the fixed point orbits are $N / T$. The coefficients $\chi^{\#}(M)$ turn out to be 1 since $N / T$ is discrete and we end up with the following formula due to Brumfiel and Madsen [BM] (see [F, VI.5] for a more detailed derivation of this formula from the general double coset theorem).

$$
\rho^{*}(T, G) \circ T(T, G)=\sum C g \text {. }
$$

The sum is over the elements of the Weyl group $W=N / T$, where $g T \in W$.

Following the approach in $[\mathbf{B M}]$ we exhibit a simple transfer proof of the following. 
THEOREM I.5 (A. BOREL).

$$
H^{*}(B G, Q) \simeq H^{*}(B T, Q)^{W}=I N V
$$

where INV is the invariants of $H^{*}(B T, Q)$ under the action of $W$. Furthermore $H^{*}(B G, \mathbf{Z}) \otimes \mathbf{Z}[1 /|W|] \simeq H^{*}(B T, \mathbf{Z})^{W} \otimes \mathbf{Z}[1 /|W|]$.

Proof. $\rho^{*}(T, G)=C g \circ \rho^{*}(T, G)$. (This follows since if $\tilde{C} g$ is the map which induces $C g\left(\left[\mathbf{F}_{0}\right.\right.$, II.3] or II.1 when $X$ equals a point), $\rho(T, G) \circ \tilde{C} g=\rho(T, G)$.) Hence the image of $\rho^{*}(T, G)$ is contained in INV. Suppose $y \in H^{*}(B G, Q)$. Then $T(T, G) \circ \rho^{*}(T, G)(y)=\chi(G / T)(y)=|W|(y)$ by Property I.1 and the fact that $\chi(G / T)=|W|$. This latter well-known fact is due to Hopf and Samelson [HS]. A similar proof appears in $\left[\mathrm{F}_{2}, \S 4\right]$. Since $|W|$ is invertible in $Q, T(T, G) \circ \rho^{*}(T, G)$ is onto $H^{*}(B G, Q)$ and $\rho^{*}(T, G)$ is injective. On the other hand let $x \in \mathrm{INV}$. Then by $\operatorname{I.4} \rho^{*}(T, G) \circ T(T, G)(x)=\Sigma C g(x)=|W|(x)$. Hence $\rho^{*}(T, G)$ is onto INV.

To prove the second statement we note that the crucial fact used is that $|W|$ is invertible in the base ring.

Definition I.6. We say that $\rho^{f}(H, G): B H^{f} \rightarrow B G^{f}$ is a finite-dimensional approximation of the fiber bundle $\rho(H, G)$ if $\rho^{f}(H, G)$ is a fiber bundle pullback of $\rho(H, G)$ by an inclusion map of a finite-dimensional subspace $B G^{f}$ into $B G$ and $B G^{f}$ and $B H^{f}$ are $C W$ complexes. For example, embed $G$ in $U(n)$ for some $n$ and let $m$ be large. Let $B G^{f}=U(n+m) / G \times U(m)$ where $G$ is viewed as being equal to its image in $U(n)$. Similarly, let $B H^{f}=U(n+m) / H \times U(m)$. Then if $\rho^{f}(H, G): B H^{f} \rightarrow B G^{f}$ is defined by

$$
u(H \times U(m)) \mapsto u(G \times U(m)) \text { for } u \in U(n+m),
$$

$\rho^{f}(H, G)$ is a finite-dimensional approximation of $\rho(H, G)$.

The following is a generalization of Theorem I.5 to arbitrary cohomology theories for finite approximations of $\rho(T, G)$.

THEOREM I.7. Let $T$ be a maximal torus in a compact Lie group $G$. Let $h$ be an arbitrary cohomology theory and let $\rho^{f}(T, G): B T^{f} \rightarrow B G^{f}$ be a finite-dimensional approximation of $\rho(T, G)$. Let $h\left(B T^{f}\right)^{W} \otimes \mathbf{Z}[1 /|W|]=I N V$ be the invariants of $h\left(B T^{f}\right)$ under the action of the Weyl group, tensored with $\mathrm{Z}[1 /|W|]$. Then

$$
(T, G) \otimes \mathrm{id}: h\left(B G^{f}\right) \otimes \mathrm{Z}[1 /|W|] \simeq I N V
$$

is an isomorphism.

Proof. The proof is similar to that given for Theorem 1.5. Let $y \in I N V$. Then by Property I.1, $T(T, G) \circ \rho^{f^{*}}(T, G)(y)=(\chi(G / T)+u)(y)$ where $u \in \tilde{h}_{s}^{0}\left(B G^{f}\right) \otimes$ $\mathbf{Z}[1 /|W|]$ has positive skeletal filtration. Since $B G^{f}$ is finite dimensional $u$ is nilpotent. Hence $\chi(G / T)+u$ is a unit in $h_{s}^{0}\left(B G^{f}\right) \otimes \mathbf{Z}[1 /|W|]$ and $\rho^{f^{\prime \prime}}(T, G)$ is injective. Since (I.4) applies to all cohomology theories it follows as in Theorem I.5 that $\rho^{f^{\prime \prime}}(T, G) \otimes$ id is onto INV.

The following well-known theorem is similar to Theorem I.5. 
THEOREM I.8. Let $G_{0}$ be a closed normal subgroup of $G$ such that $\Gamma=G / G_{0}$ is finite (e.g., $G_{0}$ could be the identity component of $\left.G\right)$. Then

$$
\rho^{*}\left(G_{0}, G\right): H^{*}(B G, Q) \simeq H^{*}\left(B G_{0}, Q\right)^{\Gamma}=I N V
$$

is an isomorphism onto the invariants. Furthermore

$$
\rho^{*}\left(G_{0}, G\right) \otimes \mathrm{id}: H^{*}(B G, \mathbf{Z}) \otimes \mathbf{Z}[1 /|\Gamma|] \stackrel{\sim}{\rightarrow} H^{*}\left(B G_{0}, \mathbf{Z}\right)^{\Gamma} \otimes \mathbf{Z}[1 /|\Gamma|]
$$

is an isomorphism.

Proof. Let $H=K=G_{0}$ in the double coset theorem. Since $G_{0}$ is normal in $G$ the double coset space is the discrete set $\Gamma$ of fixed points of the left action of $G_{0}$ on $G / G_{0}$. Since $\chi^{\#}(\mathrm{pt})=1$ and $T(H, H)=\mathrm{id}$ we have

$$
\rho^{*}\left(G_{0}, G\right) \circ T\left(G_{0}, G\right)=\sum C g
$$

where the sum is over $\Gamma$ and $g G_{0} \in \Gamma$.

By Property I.1, $T\left(G_{0}, G\right) \circ \rho^{*}\left(G_{0}, G\right)=\chi(\Gamma)=|\Gamma|$. The proof then follows exactly as in Theorem I.5.

RemarK. Since $\Gamma$ is finite this proof can be deduced from the transfer for finite coverings.

We now generalize Theorem I.8 to arbitrary cohomology theories for finite approximations of $\rho\left(G_{0}, G\right)$.

THEOREM I.10. Let $G_{0}$ be a closed normal subgroup of a compact Lie group $G$. Assume that $G / G_{0}=\Gamma$ is finite. Let $\rho^{f}\left(G_{0}, G\right): B G_{0}^{f} \rightarrow B G^{f}$ be a finite approximation of $\rho\left(G_{0}, G\right)$. Let $h\left(B G_{0}^{f}\right)^{\Gamma} \otimes \mathbf{Z}[1 /|\Gamma|]=I N V$ be the invariants of $h\left(B G_{0}^{f}\right)$ under the action of $\Gamma$ tensored with $\mathrm{Z}[1 /|\Gamma|]$. Then

$$
\rho^{*}\left(G_{0}, G\right) \otimes \mathrm{id}: h\left(B G^{f}\right) \otimes \mathbf{Z}[1 /|\Gamma|] \simeq I N V
$$

is an isomorphism for all cohomology theories $h$.

Proof. The proof is completely analogous to that of Theorem I.7. (I.9) plays the role of (I.4). We note that $\chi\left(G / G_{0}\right)=\chi(\Gamma)=|\Gamma|$.

We now prove some entirely new results.

The following formula could have been arrived at easily by Brumfiel and Madsen [BM]. Let $N$ be the normalizer of a maximal torus $T$ in $G$. Then

$$
\rho^{*}(T, G) \circ T(N, G)=\rho^{*}(T, N)
$$

for all cohomology theories.

Proof. Let $H=N$ and $K=T$ in Corollary I.3. Since the fixed point orbits of $K$ in $K|G| H$ consist of only one point $N / N$ (if $N^{g} \cap T=T$ then $g \in N$ ), the double coset formula simplifies to a single term. Since $\chi^{\#}(\mathrm{pt})=1$ and $C g=$ id when $g$ is the identity this term equals $\rho^{*}(T, N)$. We now prove the following.

TheOREM I.12 (REDUCtion to Coverings).

$$
\operatorname{Im} \rho^{*}(T, G)=\operatorname{Im} \rho^{*}(T, N) \text { for any cohomology theory. }
$$

Proof. This follows from (I.11) and the fact that $\rho^{*}(T, N) \circ \rho^{*}(N, G)=$ $\rho^{*}(T, G)$. 
Before we state the next theorem we need a definition.

Definition I.13. We say $y \in h(B H)$ is stable with respect to $\rho(H, G)$ if $\rho^{*}\left(H \cap H^{g}, H\right)(y)=\rho^{*}\left(H \cap H^{g}, H^{g}\right) C g(y)$ for all $g \in G$. We denote the stable elements of $h(B H)$ by $h(B H)^{s}=\mathcal{S}$. Let $\rho_{n}^{f}(H, G): B H_{n}^{f} \rightarrow B G_{n}^{f}, n \in \mathbf{Z}_{+}$be a filtration of $\rho(H, G)$ by finite-dimensional approximations. We use $\delta_{n}$ to denote the stable elements of $h\left(B H_{n}^{f}\right)$ with respect to $\rho_{n}^{f}(H, G)$.

The notion of stable elements has been around for a long time [CE, p. 257]. It should be thought of as a generalization of the notion of invariant elements under a group action. If $H$ is normal in $G$ the stable elements are those which are invariant under the group action of $G / H$.

Let $\tilde{C} g$ be the map which induces $C g: h(B H) \rightarrow h\left(B H^{g}\right)$ [F, II.3]. Since $\rho(H, G) \circ \tilde{C} g=\rho\left(H^{g}, G\right)$ (see [F, II.2, II.3] or this paper Definition II.1 with $X$ equal to a point) the image of $\rho^{*}(H, G)$ is contained in the stable elements. The following is similar to Property I.1 in spirit.

TheOREM I.14. Let $H$ be any closed subgroup of a compact Lie group $G$. Then if $y \in h(B H)$ is stable with respect to $\rho(H, G)$

$$
\rho^{*}(H, G) T(H, G)(y)=(\chi(G / H)+u)(y)
$$

where $u \in \tilde{h}_{s}^{0}(B H)$. We are using the fact that stable cohomotopy acts naturally on all cohomology theories.

If $h=H^{*}$ the formula is true without the error term $u$.

Proof. Let $y \in h(B H)$ be stable. By the double coset Theorem I.2

$$
\begin{aligned}
& \rho^{*}(H, G) T(H, G) y=\sum \chi^{\#}(M) T\left(H^{g} \cap H, H\right) \rho^{*}\left(H^{g} \cap H, H^{g}\right) C g(y) \\
&= \sum \chi^{\#}(M) T\left(H^{g} \cap H, H\right) \rho^{*}\left(H^{g} \cap H, H\right)(y) \\
&= \sum \chi^{\#}(M)\left(\chi\left(H / H^{g} \cap H\right)+u_{g}\right)(y) \\
& \text { by Property I.1 where } u_{g} \in \tilde{h}_{s}^{0}(B H) \\
&=\left(\left(\sum \chi^{\#}(M) \chi\left(H / H^{g} \cap H\right)\right)+\left(\sum \chi^{\#}(M) u_{g}\right)\right)(y) .
\end{aligned}
$$

Let $u=\Sigma \chi^{\#}(M) u_{g} \in \tilde{h}_{s}^{0}(B H)$. Then we have

$$
\rho^{*}(H, G) T(H, G)(y)=\left(\left(\sum \chi^{\#}(M) \chi\left(H / H^{g} \cap H\right)\right)+u\right)(y) \text {. }
$$

We claim $\Sigma \chi^{\#}(M) \chi\left(H / H^{g} \cap H\right)=\chi(G / H)$.

This follows by a calculation in singular cohomology, $H^{*}$, with integral coefficients. Let $y=\rho^{*}(H, G)(1)$. Then $y$ is stable and nontorsion. Thus $\rho^{*}(H, G) T(H, G)(y)=\Sigma \chi^{\#}(M) \chi\left(H / H^{g} \cap H\right)(y)$ since we are acting in $H^{*}$ and hence can neglect the $u_{g}$. On the other hand $\rho^{*}(H, G) T(H, G) \rho^{*}(H, G)(1)=$ $\rho^{*}(H, G) \chi(G / H)(1)=\chi(G / H)(y)$. Since $y$ is nontorsion this proves the claim and hence the theorem.

We now prove the following.

THEOREM I.15. Let $G$ be a compact Lie group, $T$ a maximal torus with normalizer $N$. Then if $h$ is any cohomology theory (even nonmultiplicative) $\rho^{f^{\prime \prime}}(N, G): h\left(B G_{n}^{f}\right) \stackrel{\sim}{\rightarrow}$ $\mathcal{S}_{n}$ is an isomorphism onto the stable elements. 
Proof. As noted previously (Definition $\operatorname{I.13)} \operatorname{Im} \rho_{n}^{f^{*}}(N, G) \subset \mathcal{S}_{n}$. Let $y \in$ $h\left(B G_{n}^{f}\right)$. Since $\chi(G / N)=1$ we have by Property I.1 that $T(N, G) \rho^{f^{*}}(N, G)(y)=$ $\left(1+w_{n}\right)(y)$ where $w_{n} \in \tilde{h}_{s}^{0}\left(B G_{n}^{f}\right)$. Let $x \in \mathcal{S}_{n}$. Then by Theorem I.14 $\rho_{n}^{f^{*}}(N, G) T(N, G)(x)=\left(1+u_{n}\right)(x)$ where $u_{n} \in \tilde{h}_{s}^{0}\left(B N_{n}^{f}\right)$. Since $B G_{n}^{f}$ and $B N_{n}^{f}$ have finite dimension and $u_{n}$ and $w_{n}$ have positive skeletal filtrations, $u_{n}$ and $w_{n}$ are nilpotent. Hence $\left(1+u_{n}\right)$ and $\left(1+w_{n}\right)$ are units in $h_{s}^{0}\left(B N_{n}^{f}\right)$ and $h_{s}^{0}\left(B G_{n}^{f}\right)$ respectively.

REMARK I.16. The extent to which Theorem I.15 is true for the map $\rho(N, G)$ : $B N \rightarrow B G$ depends on what happens to certain $\lim ^{1}$ terms that appear when passing to limits. Specifically, for $\rho_{n}^{f}(N, G), n \in \mathbf{Z}_{+}$, a filtration of $\rho(N, G)$ by finite-dimensional approximations, we have an exact sequence

$$
0 \rightarrow \lim ^{1} \mathcal{S}_{n} \rightarrow h(B G) \rightarrow \lim ^{0} \mathcal{S}_{n} \rightarrow 0
$$

if $h$ satisfies the wedge axiom [Sr]. This follows by the exact sequence for $h(B G)$, where $B G$ is filtered by the $B G_{n}^{f}$, and the prior results. We also have an exact sequence (which arises from the exact sequence for $B N$, where $B N$ is filtered by finite approximations $B N_{n}^{f}$ )

$$
0 \rightarrow\left[\lim ^{1} h\left(B N_{n}^{f}\right)\right]^{s} \rightarrow \mathcal{S} \rightarrow \lim ^{0} \mathcal{S}_{n} \rightarrow 0
$$

where $\left[\lim ^{1} h\left(B N_{n}^{f}\right)\right]^{s}$ are the stable elements of $\lim ^{1}\left(h\left(B N_{n}^{f}\right)\right)$. Hence if $\left[\lim ^{1} h\left(B N_{n}^{f}\right)\right]^{s}=\lim ^{1} \mathcal{S}_{n}$ the five lemma implies $h(B G) \simeq \delta$. This is trivially satisfied if $\lim ^{1} h\left(B N_{n}^{f}\right)=0$. This is true for all theories satisfying the Mittag-Leffler condition. In particular $H^{*}(B G) \simeq \mathcal{S}$. In general I see no reason why $\lim ^{1} \mathfrak{S}_{n}$ should equal $\left[\lim ^{1} h\left(B N_{n}^{f}\right)\right]^{s}$.

ACKNOwLEDGEMENT. I wish to thank Albrecht Dold for indicating how to generalize from the case $h=H^{*}$ to this theorem.

EXAMPLE I.17. Stable elements are in general difficult to recognize. Evens [E] calculates $\operatorname{Im} \rho^{*}(N, G)$ for integral cohomology when $G=U(n)$ which by the above theorem equals the stable elements. The answer is complicated even in this case.

We need some notation to state the next theorem. For any compact Lie group $G$ let $0 \rightarrow T \rightarrow N \stackrel{\pi}{\rightarrow} W \rightarrow 0$ be an exact sequence where $W$ is the Weyl group of $G, T$ is a maximal torus in $G$ and $N$ is the normalizer of $T$ in $G$. Let $S p$ be a Sylow $p$-subgroup of $W$.

DEFINITION I.18. Let $N p=\pi^{-1}(S p)$. Let $h\left(B G_{n}^{f}, p\right)$ be the subgroup of elements in $h\left(B G_{n}^{f}\right)$ which have order a power of $p$. Let $\left(\mathcal{S}_{n}, p\right)$ be the subgroup of elements of $\mathcal{S}_{n}$ which have order a power of $p$.

The following theorem generalizes an old result for finite groups [CE, p. 259]. (If $T$ is trivial, then $G=N=W$ and $N p=S p$.)

THEOREM I.19. $\rho^{f^{*}}(N p, G): h\left(B G_{n}^{f}, p\right) \simeq\left(\mathcal{S}_{n}, p\right)$ is an isomorphism.

Proof. The proof follows in a similar manner to that of Theorem I.15. We note that $\chi(G / N p)$ is prime to $p$.

REMARK I.20. As in Remark I.16 if $\lim ^{1} h\left(B N_{n}^{f}, p\right)=0$ and $h$ satisfies the wedge axiom, the theorem is true for $\rho(N p, G)$. Thus $H^{*}(B G, p) \stackrel{\sim}{\rightarrow}(\delta, p)$. 
II. We generalize these theorems to certain equivariant cohomology theories in this section. First we need to state and prove a generalization of the double coset theorem. It follows from [F, VI.14].

DEFINITION II.1. Let $E$ be a highly-connected paracompact and locally compact free $G$-space (as in $\left[F_{0}, I I\right]$ ) with $G$ acting on the right. For example, if $G$ is embedded in $U(n)$ let $E=U(n+m) / I_{n} \times U(m)$ where $I_{n}$ is the identity element in $U(n)$ and $m$ is large. Let $X$ be a compact and locally compact space on which $G$ acts on the left. Let $E \times X$ be the cartesian product with $G$ acting on the left by $g(e, x)=\left(e g^{-1}, g x\right)$ where $e \in E, x \in X$. Let $X_{G}=E \times_{G} X$ be the orbit space of this free action of $G$. Elements of $X_{G}$ are orbits $G(e, x)$. Let $g \in G$ and let $H$ be a subgroup of $G$. $\tilde{C} g^{X}: X_{H^{8}} \rightarrow X_{H}$ is given by $H^{g}(e, x) \rightarrow H\left(e g, g^{-1} x\right)$ and induces a conjugation $C g^{X}: h\left(X_{H}\right) \rightarrow h\left(X_{H^{g}}\right)$ for any cohomology theory $h . \rho_{X}(H, G): X_{H} \rightarrow$ $X_{G}$ is defined by $H(e, x) \mapsto G(e, x)$. Since $\rho_{X}(H, G)$ is a fibration with compact fiber and has a base space which is locally compact and paracompact, a transfer homomorphism $T_{X}(H, G): h\left(X_{H}\right) \rightarrow h\left(X_{G}\right)$ associated to the identity map of $X_{H}$ over $X_{G}$ exists [ $\left.\mathbf{D}_{1}\right] . h\left(X_{G}\right)$ is defined to be the $G$ equivariant cohomology of $X$ for the theory $h$.

REMARK. We need the various compactness conditions for $E$ and $X$ since technically the transfer is only defined in these circumstances. When generalized suitably, as it should be in the near future, $E$ can be the universal space for $B G$, i.e., a contractible free $G$-space.

If $X$ is a point, we recover the situation in $\S \mathrm{I} . X_{G}=B G, \rho_{X}(H, G)=\rho(H, G)$, $\tilde{C} g^{x}=\tilde{C} g$.

\section{THEOREM II.2.}

$$
\rho_{X}^{*}(K, G) \circ T_{X}(H, G)=\sum \chi^{\#}(M) T_{X}\left(H^{g} \cap K, K\right) \circ \rho_{X}^{*}\left(H^{g} \cap K, H^{g}\right) \circ C g^{X}
$$

where as in Property I.2 the sum is over the orbit-type manifold components $\{M\}$ of the double coset space $K|G| H$.

If $X$ is a point we recover the double coset Theorem I.2.

Proof. Consider the pullback squares, where $\pi$ by abuse of notation shall always denote the map collapsing $X$ to a point * .

$$
\begin{array}{ccccc}
\Gamma X & \stackrel{\pi}{\rightarrow} & X_{H} & \stackrel{\pi}{\rightarrow} & B H=E \times_{H} * \\
\gamma^{X} \downarrow & \rho_{X}(H, G) & \downarrow & \rho(H, G) & \downarrow \\
X_{K} & \underset{\rho_{X}(K, G)}{\rightarrow} & X_{G} & \vec{\pi} & B G=E \times_{G} *
\end{array}
$$

For any cohomology theory $h$, let $t_{\mathrm{id}}^{\Gamma X}: h(\Gamma X) \rightarrow h\left(X_{K}\right)$ be the transfer homomorphism associated to the identity map of $\Gamma X$. This map is compactly fixed (i.e., $\gamma^{X}$ is proper) since the fiber of $\gamma^{X}$ is the compact space $G / H$ [D $]$, so $t_{\text {id }}^{\Gamma X}$ exists. We have by naturality $\left[\mathrm{F}_{0}\right.$, II.13] $\rho_{X}^{*}(K, G) \circ T_{X}(H, G)=t_{\text {id }}^{\Gamma X} \circ l_{X}^{*}$. Since $\rho_{X}(H, G)$ is a pullback of $\rho(H, G)$ so is $\gamma^{X}$. Moreover $\pi \circ \rho_{X}(K, G)$ factors through $B K$. Hence $\gamma^{X}$ is also a pullback in the following squares (see [ $\mathbf{F}_{0}$, II.18]). 


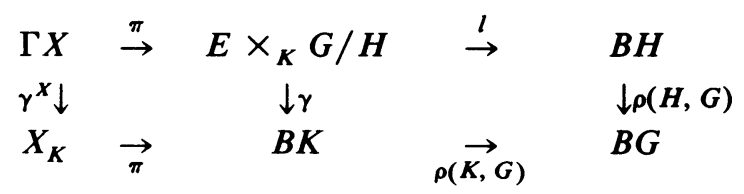

Hence $\gamma^{X}$ is a fiber bundle with structure group $K$ acting differentiably on the fiber $G / H$ on the left.

The rest of the proof follows closely the proof of the double coset theorem in $\left[F_{0}\right]$. We have by $\left[F_{0}\right.$, V.14]

$$
t_{\mathrm{id}}^{\Gamma X}=\sum \chi^{\#}(M) t_{\mathrm{id}}^{\hat{q}} k_{X}^{*}
$$

where the sum is over all orbit-type manifold components $\{M\}$ of $K|G| H . g \in G$ is a representative of $M . \hat{q} \subset \Gamma X$ is the total space of the subbundle of $\gamma^{X}$ corresponding to the $K$-orbit $K g H$ (this is the pullback of $E \times{ }_{K} K g H$ ). $k_{X}: \hat{q} \rightarrow \Gamma X$ is the inclusion. $\chi^{\#}(M)$ is the internal Euler characteristic (Property I.2), and $t_{\text {id }}^{\hat{q}}$ : $h(\hat{q}) \rightarrow h\left(X_{K}\right)$ is the transfer associated to the identity map of $\hat{q}$ over $X_{K}$.

Since there exists a homeomorphism $f: B H^{g} \cap K \rightarrow E \times{ }_{K} K g H$ [F , after V.10] over $B K$ (i.e., $\gamma \circ f=\rho\left(H^{g} \cap K, K\right)$ ) we have there exists a homeomorphism $f_{X}$ : $X_{H^{8} \cap K} \rightarrow \hat{q}$ between the pullbacks of $B H^{g} \cap K$ and $E \times_{K} K g H$ under the map $\pi$ : $X_{K} \rightarrow B K$ such that $\gamma^{X} \circ f_{X}=\rho_{X}\left(H^{g} \cap K, K\right)$. Hence by naturality [Fo, II.13] $t_{\text {id }}^{\hat{q}}=T_{X}\left(H^{g} \cap K, K\right) \circ f_{X}^{*}$. The theorem follows once we observe that $l_{X} \circ k_{X} \circ f_{X}$ $=\tilde{C} g^{X} \circ \rho_{X}\left(H^{g} \cap K, H^{g}\right)$. This follows since these maps are the pullbacks under the collapsing map $\pi: X_{H} \rightarrow B H$ of $l \circ k \circ f$ and $\tilde{C} g \circ \rho\left(H^{g} \cap K, H^{8}\right)$ and these maps agree [F, V.12].

The following theorems are direct generalizations of theorems proved in the first section of this paper (let $X$ equal a point). Their proofs are analogous. Theorem II.3 is well known [H, p. 38]. Theorems II.4 and II.5 are well known for $h=H^{*}[\mathbf{H}, \mathrm{p}$. 38] but new in the generality stated. Theorems II.6, II.8, II.9, and II.10 are completely new.

THEOREM II.3. $\rho_{X}^{*}(T, G): H^{*}\left(X_{G}, Q\right) \simeq H^{*}\left(X_{T}, Q\right)^{W}$ is an isomorphism where $T$ is a maximal torus of $G$ and $W$ is the Weyl group. Similarly

$$
\rho_{X}^{*}(T, G) \otimes \mathrm{id}: H^{*}\left(X_{G}, \mathbf{Z}\right) \otimes \mathbf{Z}\left[\frac{1}{|W|}\right] \simeq H^{*}\left(X_{T}\right)^{W} \otimes \mathbf{Z}\left[\frac{1}{|W|}\right]
$$

is an isomorphism.

This generalizes Theorem I.5.

THEOREM II.4. If $E$ and $X$ are finite $C W$ complexes, then

$$
\rho_{X}^{*}(T, G) \otimes \mathrm{id}: h\left(X_{G}\right) \otimes \mathbf{Z}\left[\frac{1}{|W|}\right] \simeq h\left(X_{T}\right)^{W} \otimes \mathbf{Z}\left[\frac{1}{|W|}\right]
$$

is an isomorphism onto the invariants of $h\left(X_{T}\right)$ tensored with $\mathrm{Z}[1 /|W|]$ where $h$ is any cohomology theory.

This generalizes Theorem I.7. 
TheOREM II.5. Let $G_{0}$ be a closed normal subgroup of $G$ such that $G / G_{0}=\Gamma$ is finite (e.g., $G_{0}$ could be the identity component of $G$ ). Let $E$ and $X$ be finite $C W$ complexes. Then

$$
\rho_{X}^{*}\left(G_{0}, G\right) \otimes \mathrm{id}: h\left(X_{G}\right) \otimes \mathbf{Z}\left[\frac{1}{|\Gamma|}\right] \simeq h\left(X_{G_{0}}\right)^{\Gamma} \otimes \mathbf{Z}\left[\frac{1}{|\Gamma|}\right]
$$

is an isomorphism.

This generalizes Theorem I.10.

THEOREM II.6. $\operatorname{Im} \rho_{\mathrm{X}}^{*}(T, G)=\operatorname{Im} \rho_{X}^{*}(T, N)$ for any cohomology theory.

This generalizes Theorem I.12.

We need a definition to state the analogues of Theorems I.14, I.15, I.19. Their proofs are analogous.

Definition II.7. An element $y \in h\left(X_{H}\right)$ is stable with respect to $\rho_{X}(H, G)$ if $\rho_{X}^{*}\left(H \cap H^{g}, H^{g}\right) C g^{X}(y)=\rho_{X}^{*}\left(H \cap H^{g}, H\right)(y)$ for all $g \in G$. Denote the stable elements by $\mathcal{S}_{X}$.

TheORem II.8. Let $y \in h\left(X_{H}\right)$ be stable with respect to $\rho_{X}(H, G)$. Then $\rho_{X}^{*}(H, G) \circ T_{X}(H, G)(y)=(\chi(G / H)+u)(y)$ where $u \in \tilde{h}_{s}^{0}\left(X_{H}\right)$ and has positive skeletal filtration in stable cohomotopy.

This generalizes Theorem I.14.

TheOREM II.9. Suppose $X$ and $E$ are finite-dimensional $C W$ complexes. Then

$$
\rho_{X}^{*}(N, G): h\left(X_{G}\right) \stackrel{\sim}{\rightarrow} h\left(X_{N}\right)^{s}=\mathcal{S}_{X} .
$$

This generalizes Theorem I.15.

Let $N p$ be defined as in Definition I.18. Let $\left(\delta_{X}, p\right)$ be the subgroup of stable elements of $h\left(X_{N p}\right)$ which have order a power of $p$.

Theorem II.10. Suppose $X$ and $E$ are finite $C W$ complexes. Then $\rho_{X}^{*}(N p, G)$ : $h\left(X_{G}, p\right) \simeq h\left(X_{N p}, p\right)^{s}=\left(\delta_{X}, p\right)$ is an isomorphism of the elements of $h\left(X_{G}\right)$ which have order a power of $p$ onto the stable elements of $h\left(X_{N p}\right)$ which have order a power of $p$.

This generalizes Theorem I.19.

\section{REFERENCES}

[BG] J. C. Becker and D. Gottlieb, The transfer for fibrations and duality, Compositio Math. 33 (1976), 107-133.

[BM] G. Brumfiel and I. Madsen, Evaluation of the transfer and the universal surgery classes, Invent. Math. 32 (1976), 133-169.

[CE] H. Cartan and S. Eilenberg, Homological algebra, Princeton Univ. Press, Princeton, N. J., 1956.

[C.] A. Dold, The fixed point transfer of fibre-preserving maps, Math. Z. 148 (1976), 215-244.

$\left[\mathbf{D}_{1}\right]$, Transfert des points fixes d' une famille continue d'applications, C. R. Acad. Sci. Paris Sér. A 278 (1974), 1291-1293.

[D $\mathrm{D}_{2} \ldots$, The fixed point index of fibre-preserving maps, Invent. Math. 25 (1974), 281-297.

[Dy] E. Dyer, Cohomology theories, Benjamin, New York, 1969.

[E] L. Evens, On the Chern classes of representations of finite groups, Trans. Amer. Math. Soc. 115 (1965), 180-193. 
[Fo] M. Feshbach, The transfer and compact Lie groups, Trans. Amer. Math. Soc. 251 (1979), 139-169.

$\left[F_{1}\right]$, The transfer and compact Lie groups, Bull. Amer. Math. Soc. 83 (1977), $372-374$.

$\left[\mathrm{F}_{2}\right] \longrightarrow$, The transfer and characteristic classes, Proc. Northwestern Conf. on Geometric Applications of Homotopy Theory, March 1977, Lecture Notes in Math., vol. 657, Springer-Verlag, Berlin and New York, 1978, pp. 156-162.

[HS] H. Hopf and H. Samelson, Ein Satz über die Wirkungsraume geschlossener Lie'scher Gruppen, Comment Math. Helv. 13 (1940), 240-251.

[H] W.-Y. Hsiang, Cohomology theory of topological transformation groups, Springer-Verlag, Berlin, 1975.

[Sr] R. M. Switzer, Algebraic topology-homotopy and homology, Springer-Verlag, Berlin, 1975.

Department of Mathematics, Northwestern University, Evanston, Ilunois 60201

Current address: School of Mathematics, University of Minnesota, Minneapolis, Minnesota 55455 